\title{
ON THE DETECTABILITY OF DUAL JETS FROM BINARY BLACK HOLES
}

\author{
Philipp Moesta ${ }^{1,2}$, Daniela Alic ${ }^{1}$, Luciano Rezzolla $^{1,3}$, Olindo Zanotti $^{1}$, and Carlos Palenzuela ${ }^{3,4}$ \\ ${ }^{1}$ Max-Planck-Institut für Gravitationsphysik, Albert-Einstein-Institut, Potsdam-Golm, Germany \\ 2 TAPIR, MC 350-17, California Institute of Technology, Pasadena, CA 91125, USA \\ ${ }^{3}$ Department of Physics and Astronomy, Louisiana State University, Baton Rouge, LA, USA \\ ${ }^{4}$ Canadian Institute for Theoretical Astrophysics, Toronto, Ontario M5S 3H8, Canada \\ Received 2012 February 23; accepted 2012 March 7; published 2012 March 30
}

\begin{abstract}
We revisit the suggestion that dual jets can be produced during the inspiral and merger of supermassive black holes when these are immersed in a force-free plasma threaded by a uniform magnetic field. By performing independent calculations of the late inspiral and merger, and by computing the electromagnetic (EM) emission in a way which is consistent with estimates using the Poynting flux, we show that a dual-jet structure is present but energetically subdominant with respect to a non-collimated and predominantly quadrupolar emission, which is similar to the one computed when the binary is in electrovacuum. While our findings set serious restrictions on the detectability of dual jets from coalescing binaries, they also increase the chances of detecting an EM counterpart from these systems.
\end{abstract}

Key words: binaries: close - galaxies: jets - gravitation - magnetic fields - plasmas - relativistic processes

Online-only material: color figures

\section{INTRODUCTION}

The inspiral and merger of supermassive black holes (BHs) will represent a secure source for the planned space-borne gravitational-wave (GW) detectors. Together with the GW signal, this process is expected to be accompanied either before (Bode et al. 2012; Farris et al. 2011) or after (Rossi et al. 2010; Zanotti et al. 2010) the merger by the emission of electromagnetic (EM) radiation, thus providing a perfect example of multimessenger astronomy. Should a "simultaneous" detection take place, this would not only help to localize the GW source and provide its redshift, but also to address a number of questions in astrophysics and cosmology (Haiman et al. 2009; Sesana et al. 2011).

As the merger between two galaxies takes place and the $\mathrm{BHs}$ get closer, a circumbinary accretion disk is expected to form. Because the radiation-reaction timescale over which the binary evolves is much longer than the accretion timescale, the disk will slowly follow the binary as its orbit shrinks. However, as the binary separation becomes $\sim 10^{5} M$, where $M$ is the mass of the binary, the radiation-reaction timescale reduces considerably, becoming smaller than the accretion one. When this happens, the disk evolution disconnects from the binary, the accretion rate reduces, and the binary performs its final orbits in an inner region poor of gas (Armitage \& Natarajan 2002; Liu et al. 2003).

This basic picture represents the astrophysical backdrop of a simple model which has been used by a number of authors to model the EM emission from the BH binary. More specifically, assuming that the disk is threaded by a coherent and largescale magnetic field which is anchored to the disk, this will also permeate the "evacuated" region where the binary is rapidly shrinking and provide an effective way of coupling the binary's orbital motion to the generation of an EM signal. This scenario has been considered both for spacetimes in electrovacuum (EV) (Palenzuela et al. 2009, 2010; Mösta et al. 2010) and for spacetimes filled by a tenuous plasma in the force-free (FF) approximation (Palenzuela et al. 2010a, 2010b; Neilsen et al. 2011). Mösta et al. (2010), in particular, have considered a series of spinning $\mathrm{BH}$ binaries and studied the dependence of the gravitational and EM signals with different spin configurations. All in all, it was found that EM radiation in the lowest $\ell=2, m=2$ multipole accurately reflects the gravitational one. Furthermore, the efficiency of the energy emission in EM waves was found to scale quadratically with the total spin and to be $\sim 13$ orders of magnitude smaller than the one in GWs for realistic magnetic fields. However, the prospects of detecting an EM counterpart have become larger when it was pointed out in Palenzuela et al. (2010b) that, during the inspiral in an FF plasma, a dual-jet structure forms, as a generalization of the Blandford-Znajek process (Blandford \& Znajek 1977) to orbiting BHs. Under these conditions, the EM energy can accelerate electrons and lead to synchrotron radiation.

We extend here the investigations made in Mösta et al. (2010) by considering the evolution in an FF regime and properly computing the EM emission as the net result of ingoing and outgoing radiation. We confirm in this way the presence of a dual-jet structure, but also find that this is energetically subdominant with respect to a non-collimated and predominantly quadrupolar emission, at least during the late inspiral and merger we have considered. Hence, if the simplified scenario considered here is realized in astrophysical configurations, it will be difficult to reveal the emission from the dual jets, but it will also be easier to reveal that an EM counterpart to binary $\mathrm{BH}$ mergers can be detected.

\section{METHODOLOGY}

As mentioned above, we assume the magnetic field to be anchored to the disk, whose inner edge is at a distance of $\sim 10^{3} \mathrm{M}$ and is effectively outside of our computational domain, while the initial binary separation is only $D=8 \mathrm{M}$. Although the largescale magnetic field is poloidal, it is set to be initially uniform within the computational domain, i.e., $B^{i}=\left(0,0, B_{0}\right)$ with $B_{0} \sim 10^{-4} / M$. We note that although astrophysically large, the initial magnetic field has an EM energy several orders of magnitude smaller than the gravitational field one, so that the EM fields can be treated as test fields and all the results for the 
luminosity can scale quadratically with $B_{0}$. The electric field $E^{i}$ is initially zero, but it rapidly reaches a consistent solution (Mösta et al. 2010). For BH initial data, we consider binaries with equal masses but with two different spin configurations: namely, the $s_{0}$-binary (both $\mathrm{BHs}$ are nonspinning) and the $s_{6}$-binary (both BHs have spins aligned with the orbital angular momentum with $\left(J / M^{2}\right)_{1}=a_{1}=a_{2}=0.6$; see Rezzolla et al. 2008; Reisswig et al. 2009 for details). These two configurations allow us to study both the contributions coming from the binary's orbital motion, and also those related to the spins of the two BHs. The setup for the numerical grids used in the simulations consists of nine levels of mesh refinement with a fine resolution of $\Delta x / M=0.025$. The wave-zone grid has a resolution of $\Delta x / M=1.6$ and extends from $r=24 M$ to $r=180 M$, while the outer (coarsest) grid extends to $819.2 M$. In our implementation of the FF equations, we solve the same set of equations in Palenzuela et al. (2010a) but do not enforce "by-hand" the FF condition. Rather, we implement a damping scheme which drives the solution to satisfy the FF condition and avoids inconsistent EM fields; we will present our approach in D. Alic et al. (2011, in preparation).

The calculation of the EM and gravitational radiation generated during the inspiral, merger, and ringdown is arguably the most important aspect of this work, as it allows us to establish what are the characteristics of the emissions in two channels. For the GW sector, we compute the emission via the Newman-Penrose (NP) curvature scalar $\Psi_{4}$ as detailed in Pollney et al. (2007) and Mösta et al. (2010). In practice, we define an orthonormal basis in the three space $(\hat{\boldsymbol{r}}, \hat{\boldsymbol{\theta}}, \hat{\boldsymbol{\phi}})$, with poles along $\hat{z}$. Using the normal to the slice as timelike vector $\hat{\boldsymbol{t}}$, we construct the null orthonormal tetrad $\{\boldsymbol{l}, \boldsymbol{n}, \boldsymbol{m}, \overline{\boldsymbol{m}}\}$ with the bar indicating a complex conjugate

$$
\boldsymbol{l}=\frac{1}{\sqrt{2}}(\hat{\boldsymbol{t}}+\hat{\boldsymbol{r}}), \quad \boldsymbol{n}=\frac{1}{\sqrt{2}}(\hat{\boldsymbol{t}}-\hat{\boldsymbol{r}}), \quad \boldsymbol{m}=\frac{1}{\sqrt{2}}(\hat{\boldsymbol{\theta}}+i \hat{\boldsymbol{\phi}})
$$

by means of which we project the Weyl curvature tensor $C_{\alpha \beta \gamma \delta}$ to obtain $\Psi_{4}=C_{\alpha \beta \gamma \delta} n^{\alpha} \bar{m}^{\beta} n^{\gamma} \bar{m}^{\delta}$. For the EM sector, instead, we use two equivalent approaches to cross-validate our measures. The first one uses the NP scalars $\Phi_{0}$ (for the ingoing EM radiation) and $\Phi_{2}$ (for the outgoing EM radiation), defined using the same tetrad (Teukolsky 1973)

$$
\Phi_{0} \equiv F^{\mu \nu} l_{\nu} m_{\mu}, \quad \Phi_{2} \equiv F^{\mu \nu} \bar{m}_{\mu} n_{v}
$$

It is always useful to remark that, by construction, quantities such as $\Psi_{4}, \Phi_{0}, \Phi_{2}$ are well defined only at very large distances from the sources. Any measure of these quantities in the strongfield region risks being incorrect. As an example, the EM energy flux does not show the expected $1 / r^{2}$ scaling when $\Phi_{0}, \Phi_{2}$ are measured at distances of $r \simeq 20 M$ (Palenzuela et al. 2010a, $2010 b$ ), which is instead reached only for $r \gtrsim 100 \mathrm{M}$. As we will show later, this results in a considerable difference in the estimate of the non-collimated EM emission.

Since our choice of having a uniform magnetic field within the computational domain has a number of drawbacks (e.g., nonzero initial $\left.\Phi_{2}, \Phi_{0}\right)$, great care has to be taken when measuring the EM radiation. Fortunately, we can exploit the linearity in the Maxwell equations to distinguish the genuine emission induced by the presence of the $\mathrm{BH}(\mathrm{s})$ from the background emission. Following Teukolsky (1973), we compute the total EM luminosity as a surface integral across a 2-sphere at a large distance

$$
L_{\mathrm{EM}}=\lim _{r \rightarrow \infty} \int r^{2}\left(\left|\Phi_{2}-\Phi_{2, \mathrm{~B}}\right|^{2}-\left|\Phi_{0}-\Phi_{0, \mathrm{~B}}\right|^{2}\right) d \Omega,
$$

where $\Phi_{2, \mathrm{~B}}$ and $\Phi_{0, \mathrm{~B}}$ are the values of the background scalars induced by the asymptotically uniform magnetic field solution in the time-dependent spacetime produced by the binary BHs. The choice of $\Phi_{2, \mathrm{~B}}$ and $\Phi_{0, \mathrm{~B}}$ will be crucial to measure correctly the radiative EM emission.

The background values of the NP scalars $\Phi_{2, \mathrm{~B}}, \Phi_{0, \mathrm{~B}}$ to be used in Equation (3) are themselves time dependent and cannot be distinguished, at least a priori, from the purely radiative contributions. A first prescription takes the background values to be time independent, and those at the initial time

$$
\Phi_{2, \mathrm{~B}}=\Phi_{2}(t=0), \quad \Phi_{0, \mathrm{~B}}=\Phi_{0}(t=0) \text {. }
$$

A second prescription involves instead the removal of those multipole components of the NP scalars which are not radiative, namely, all those associated with the $m=0$ multipoles

$$
\Phi_{2, \mathrm{~B}}=\left(\Phi_{2}\right)_{\ell, m=0}, \quad \Phi_{0, \mathrm{~B}}=\left(\Phi_{0}\right)_{\ell, m=0},
$$

where $\left(\Phi_{2,0}\right)_{\ell, m=0}$ refer to the $m=0$ modes of the multipolar decomposition of $\Phi_{2,0}(\ell \leqslant 8$ is sufficient to capture most of the background). Note that, because the $m=0$ background is essentially time independent (after the initial transient), the choice, Equation (5), is effectively equivalent to considering as background the final values of the NP scalars as computed in an EV calculation of the same binary system.

While apparently different, expressions (4) and (5) lead to very similar estimates and, more importantly, they have a simple interpretation in terms of their corresponding measures. We recall, in fact, that the EM luminosity is customarily computed via the integral over a 2-sphere of the Poynting flux $S_{i}=\sqrt{\gamma} \epsilon_{i j k} E^{j} B^{k}$, which is again just the flux of the stressenergy tensor as measured now by observers on the spatial hypersurface. Of course, also, this measure is adequate only far from the binary and it suffers equally from a background non-radiative contribution. However, because of the linearity in the Maxwell equations, the non-radiative contributions can also be removed by introducing background values of the EM fields $E_{\mathrm{B}}^{j}, B_{\mathrm{B}}^{j}$ and computing the Poynting vector as $S_{i}=\sqrt{\gamma} \epsilon_{i j k}\left(E^{j}-E_{\mathrm{B}}^{j}\right)\left(B^{k}-B_{\mathrm{B}}^{k}\right)$. In this context, then, expressions (4) and (5) correspond, respectively, to setting $E_{\mathrm{B}}^{k}=0, B_{\mathrm{B}}^{k}=B^{k}(t=0)$ and $E_{\mathrm{B}}^{k}=E_{\ell, m=0}^{k}, B_{\mathrm{B}}^{k}=B_{\ell, m=0}^{k}$. We have verified that the measures of the EM luminosity obtained using Equation (4) or (5) reproduce well the corresponding ones obtained with the Poynting flux. As a final remark, we note that the EM flux in Equation (3) is not always positive everywhere on the 2-sphere. The negative contributions, however, average to zero over one orbit and do not represent a radiative field. This point was remarked in Palenzuela et al. (2010), where a toy model within the membrane paradigm was used for the binary.

\section{RESULTS}

Using expression (3) with either prescription (4) or (5), we find that the EM radiation generated during the late inspiral and merger contains a dual-jet structure, but also that the total energy flux is dominated by a non-collimated emission of quadrupolar nature. This is shown in Figure 1, which reports snapshots at different times of the EM energy flux on a 

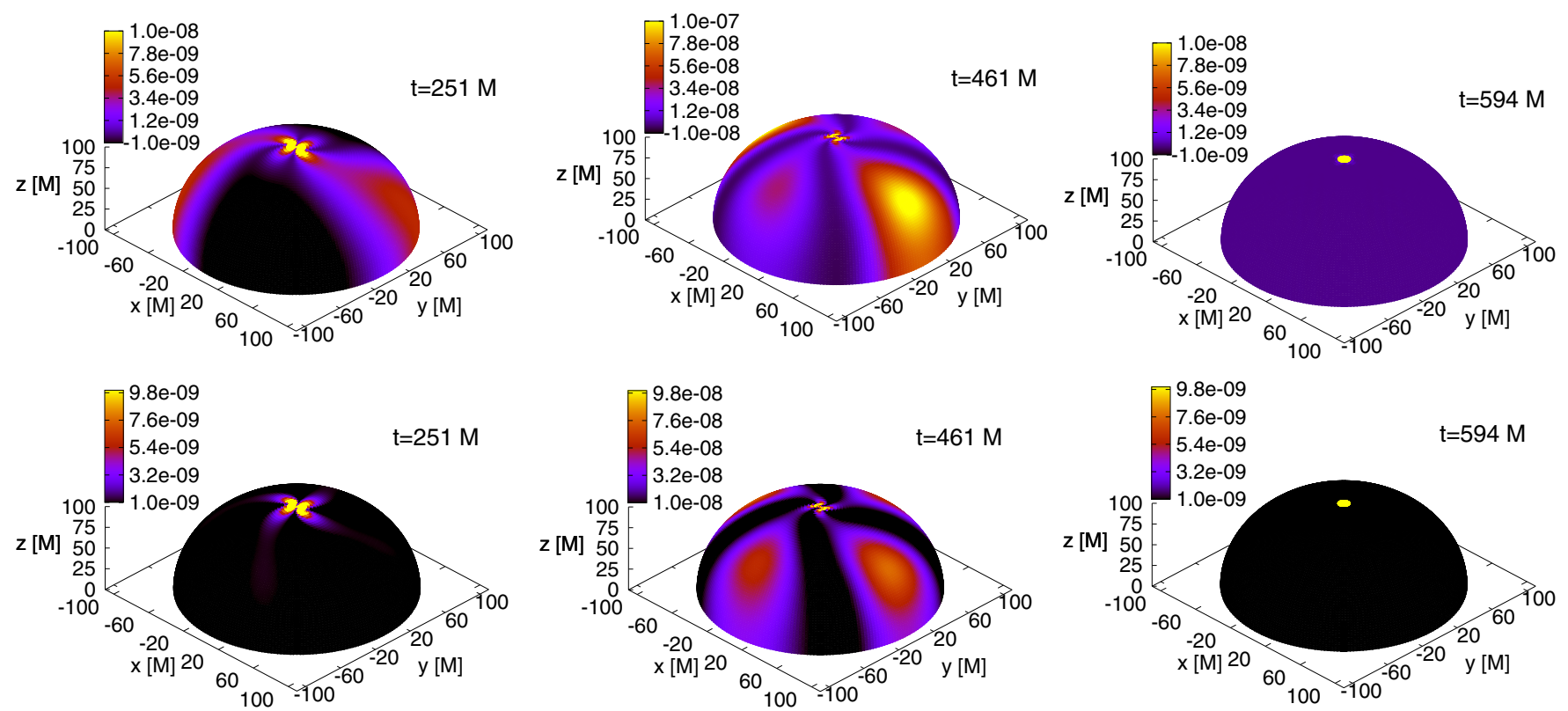

Figure 1. Snapshots of the EM energy flux during the inspiral and merger of the binary $s_{0}$. The top and bottom rows show the fluxes as measured via Equation (3) with condition Equation (4) and via Equation (5), respectively. Note that Equation (4) yields negative values which will cancel out when integrated over one orbit. (A color version of this figure is available in the online journal.)

2-sphere of radius $r=100 M$ for the $s_{0}$-binary. The top row shows the fluxes as computed with Equation (4) and with a (nonradiative) component that enhances two lobes of the signal and decreases the other two (the first, second, and third panels refer to the inspiral, merger, and ringdown stages, respectively). Although there are regions with negative values which will average to zero over one orbit, the corresponding EM fields could nevertheless induce motion in the plasma and secondary radiation which we cannot account for here. The second row refers to fluxes as computed with Equation (5), where the time-dependent background solution is almost completely subtracted, so that only the (positive) radiative part remains, showing a fairly symmetric four-lobe structure. Within both approaches, the extended quadrupolar distribution is accompanied by the presence of dual jets during the inspiral, and of a single jet from the spinning merged BH. The energy flux in the jets is essentially the same in the two cases (both during the inspiral and after the merger), but the energy flux in the non-collimated part is different. In spite of this, the total luminosities are very similar, as most of the differences cancel when integrated over one orbit (see Figure 3). However, because the Poynting-flux structure is different in the two measures (Equation (4) has a non-radiative part missing in Equation (5)), it could lead to a different secondary emission as the EM fields interact with the plasma; this emission cannot be investigated within an FF approach but deserves attention.

The corrections introduced by the spins of the BHs are shown in Figure 2, which reports map views (i.e., projections on the $(x, y)$ plane) of the EM emission for the binaries $s_{0}$ and $s_{6}$, as computed through Equation (5). Both the collimated and the non-collimated emission are very similar, although there is a $50 \%$ enhancement of the radiation in the spinning case, both in the total and in the collimated emission. This is because most of the radiation is produced by the interaction between the $\mathrm{BH}$ orbital motion and the background magnetic field. Indeed, we find the emission in the EV evolution to be comparable to the FF one (this is different from what was reported in Palenzuela et al. 2010a, 2010b).
A more quantitative assessment of the different contributions is shown in Figure 3, which reports the evolution of the EM luminosity of the binary $s_{0}$ (left panel) and of the spinning binary $s_{6}$ (right panel). Shown with (blue) solid lines is the total luminosity as computed through Equation (5), while the (red) dashed lines refer to the luminosity integrated over a polar cap with a half-opening angle of $5^{\circ}$, and thus representative of the emission from the two jets. Also shown with (magenta) dotted lines are the luminosities as computed through Equation (4): the differences are small and hardly visible for the collimated part.

An accurate measure of the evolution of the collimated/noncollimated components is crucial to predict the properties of the system when the two BHs are widely separated. This, in turn, requires a reliable disentanglement of the collimated emission from the non-collimated one and from the background. The noncollimated emission measured with Equation (5) matches well the growth expected if the EM emission is mostly quadrupolar and hence with a dependence that is the same as the GW one and, at the lowest order, scales as $L_{\mathrm{EM}}^{\text {non-coll }} \approx \Omega^{10 / 3}$ (Palenzuela et al. 2010; Mösta et al. 2010). On the other hand, a smaller scaling is found when the approach from Equation (4) is adopted; at present it is difficult to determine which one is the most reliable scaling. At the same time, the frequency evolution for the collimated emission coincides in the two approaches, but it does not follow the scaling $L_{\mathrm{EM}}^{\text {coll }} \approx \Omega^{2 / 3}$ suggested in Palenzuela et al. (2010b) for boosted BHs; rather, it scales as $L_{\mathrm{EM}}^{\text {coll }} \lesssim \Omega^{5 / 3-6 / 3}$. We suspect the accelerated motion of the BHs to be behind this difference and longer simulations are needed to draw robust conclusions.

Impact on detectability. Assessing the detectability of the EM emission discussed above is, of course, of great importance and using the results of Palenzuela et al. (2010a, 2010b), Kaplan et al. (2011) have estimated that the short timescales associated with the merger will limit the detectability in the radio band to less than $1 \mathrm{yr}^{-1}$. As reported in Figure 3, the peak of the collimated emission is $\sim 100$ times smaller than that of the total emission, making the detection of the dual jets at the merger 

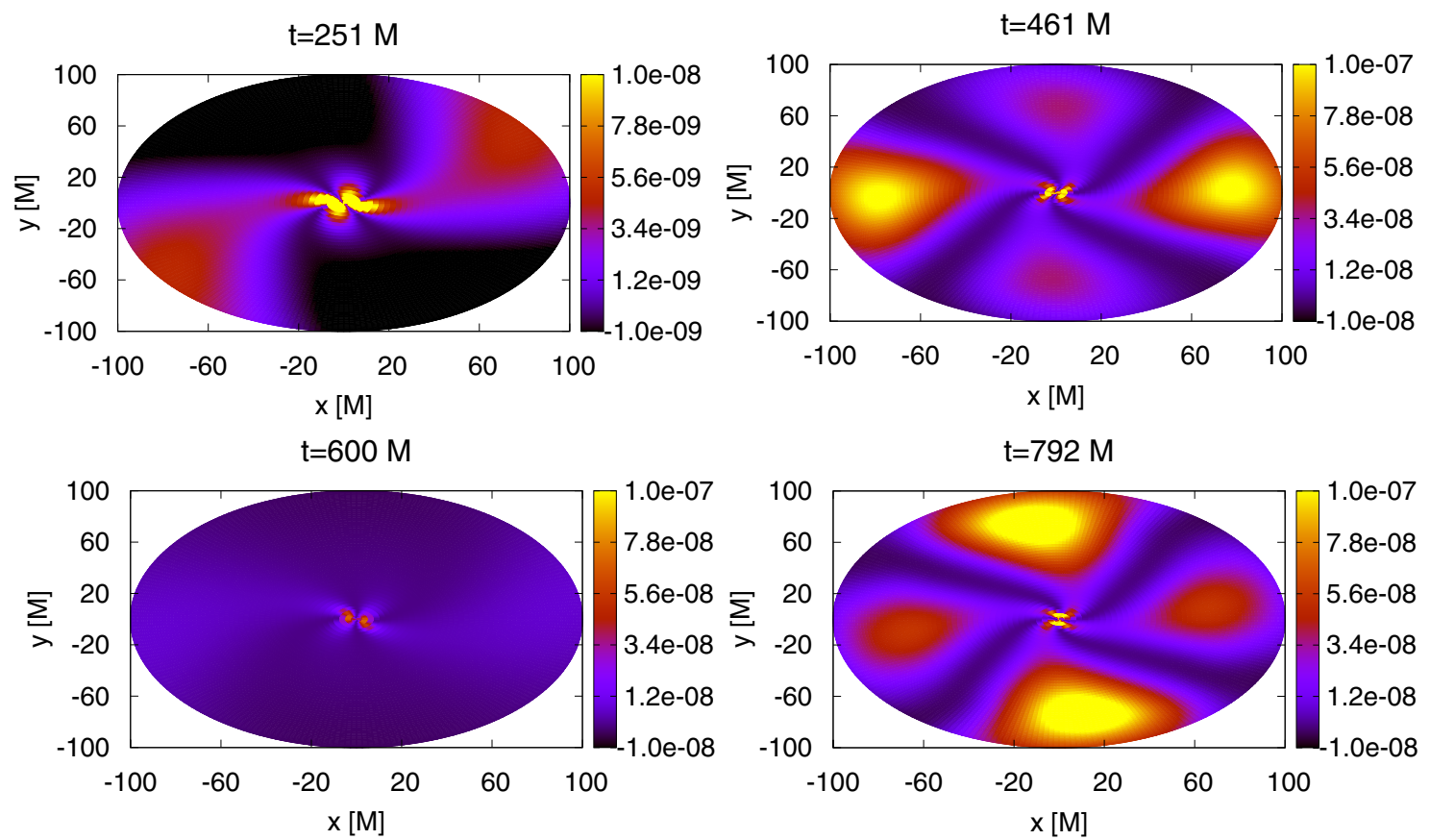

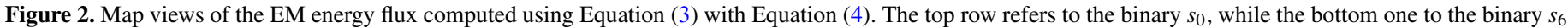
and has a larger emission from the dual jets.

(A color version of this figure is available in the online journal.)

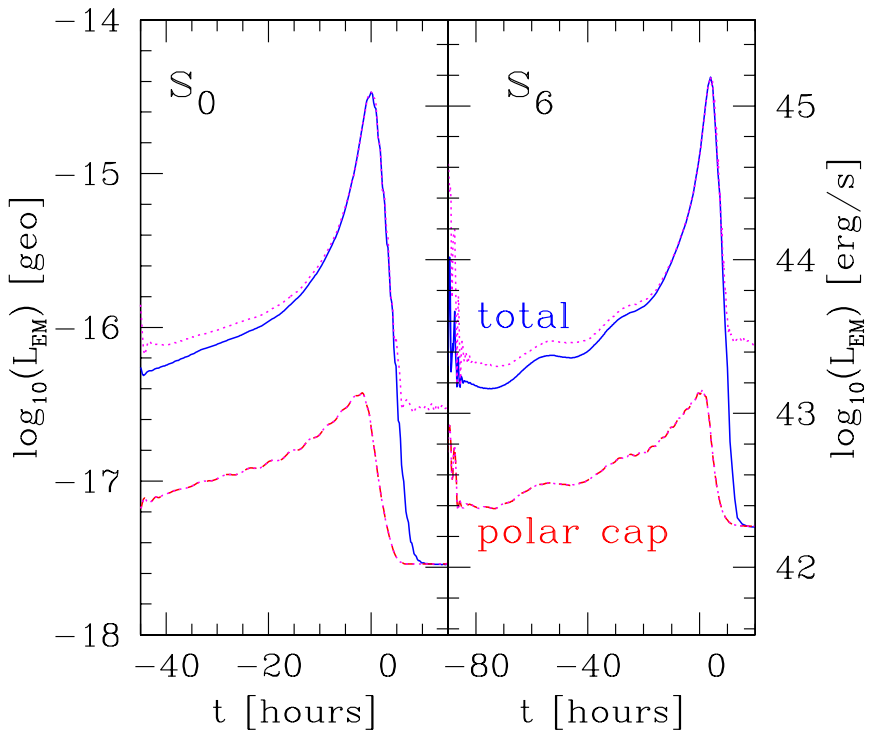

Figure 3. Evolution of the EM luminosity at $100 \mathrm{M}$ for the binary $s_{0}$ (left) and for the spinning binary $s_{6}$ (right), when $M=10^{8} M_{\odot}$ and $B_{0}=10^{4} \mathrm{G}$. Using (5), (blue) solid lines show the total luminosity, while (red) dashed lines refer to the luminosity in a polar cap of $5^{\circ}$. Shown with (magenta) dotted lines are the measures with (4); note the presence of a small eccentricity for the binary $s_{6}$.

(A color version of this figure is available in the online journal.)

unlikely. Unfortunately, even if the energy flux is $\sim 8-2$ times larger near the jets, the lack of knowledge about the Lorentz factor of the reprocessed plasma does not allow us to say whether the beaming in the jet will be larger than that in the extended emission and thus help its detection. That said, because the total luminosity at merger is $\sim 100$ times larger than in Palenzuela et al. (2010b) (mainly because we measure it at large distances where it has approximately reached its asymptotic value), the detection should be more likely overall if most of the assumptions in Kaplan et al. (2011) are verified.
In addition, the dual jets emission could be dominant in the early inspiral (especially if the BHs are spinning). Assuming the scalings for the collimated and non-collimated emissions are different and go, respectively, like $\Omega^{10 / 3}$ and $\Omega^{6 / 3}\left(\Omega^{5 / 3}\right)$, then the two components of the luminosity would become of the same order at a separation of $\sim 16(24) M$, but obviously smaller (the collimated will be smaller by a factor $\sim 10$ ). Determining more precisely when and if this happens requires an accurate frequency scaling which is not available yet. As a note of caution, we stress that luminosities $L_{\mathrm{EM}} \sim 10^{45} \mathrm{erg} \mathrm{s}^{-1}$ are also typical of radio-loud galaxies, and the determination of an EM counterpart can be challenging if such sources are near the candidate event.

\section{CONCLUSIONS}

We have investigated the suggestion that dual jets can be produced during the inspiral and merger of supermassive BHs immersed in an FF plasma threaded by a uniform magnetic field. We have found that the energy flux does have a dualjet structure, but is predominantly quadrupolar, with the noncollimated emission being about 10-100 times larger than the collimated one. Our findings set restrictions on the detectability of dual jets from coalescing $\mathrm{BH}$ binaries, but also increase the chances of detecting an EM counterpart for astrophysical conditions similar to those in this simplified scenario.

We are grateful to J.-L. Jaramillo for discussions and to L. Lehner for suggesting an approach similar to Equation (4). We also thank A. Sesana, B. Ahmedov, and V. Morozova. This work was supported in part by the DFG grant SFB/Transregio 7; the computations were made at the AEI and on TERAGRID (TG-MCA02N014).

\section{REFERENCES}

Armitage, P. J., \& Natarajan, P. 2002, ApJ, 567, L9

Blandford, R. D., \& Znajek, R. L. 1977, MNRAS, 179, 433 
Bode, T., Bogdanović, T., Haas, R., et al. 2012, ApJ, 744, 45

Farris, B. D., Liu, Y. T., \& Shapiro, S. L. 2011, Phys. Rev. D, 84, 024024

Haiman, Z., Kocsis, B., Menou, K., Lippai, Z., \& Frei, Z. 2009, Class. Quantum Grav., 26, 094032

Kaplan, D. L., O'Shaughnessy, R., Sesana, A., \& Volonteri, M. 2011, ApJ, 734, L37

Liu, F., Wu, X., \& Cao, S. 2003, MNRAS, 340, 411

Mösta, P., Palenzuela, C., Rezzolla, L., et al. 2010, Phys. Rev. D, 81, 064017

Neilsen, D., Lehner, L., Palenzuela, C., et al. 2011, Proc. Nat. Acad. Sci., 108, 12641

Palenzuela, C., Anderson, M., Lehner, L., Liebling, S. L., \& Neilsen, D. 2009, Phys. Rev. Lett., 103, 081101
Palenzuela, C., Garrett, T., Lehner, L., \& Liebling, S. L. 2010a, Phys. Rev. D, 82,044045

Palenzuela, C., Lehner, L., \& Liebling, S. L. 2010b, Science, 329, 927

Palenzuela, C., Lehner, L., \& Yoshida, S. 2010, Phys. Rev. D, 81, 084007

Pollney, D., Reisswig, C., Rezzolla, L., et al. 2007, Phys. Rev. D, 76, 124002

Reisswig, C., Husa, S., Rezzolla, L., et al. 2009, Phys. Rev. D, 80, 124026

Rezzolla, L., Dorband, E. N., Reisswig, C., et al. 2008, ApJ, 679, 1422

Rossi, E. M., Lodato, G., Armitage, P. J., Pringle, J. E., \& King, A. R. 2010, MNRAS, 401, 2021

Sesana, A., Gair, J., Berti, E., \& Volonteri, M. 2011, Phys. Rev. D, 83, 044036 Teukolsky, S. A. 1973, ApJ, 185, 635

Zanotti, O., Rezzolla, L., Del Zanna, L., \& Palenzuela, C. 2010, A\&A, 523, A8 